Vulnerabilidade e variações de curto prazo da praia de Itaipu (Niterói-RJ) em resposta às mudanças nas condições de mar Eduardo de Macedo Rodrigues, André Luiz Carvalho da Silva, Leonardo Rodrigues Eccard, Carolina Pereira Silvestre e Yame

Bronze Medina Ramos

\title{
VULNERABILIDADE E VARIAÇÕES DE CURTO PRAZO DA PRAIA DE ITAIPU (NITERÓI-RJ) EM RESPOSTA ÀS MUDANÇAS NAS CONDIÇÕES DE MAR
}

\section{Vulnerability and short-term variations of Itaipu beach (Niterói-RJ) in response to changes in sea conditions}

\author{
Eduardo de Macedo Rodrigues \\ Graduado em Geografia pela Faculdade de Formação de Professores da FFP/UERJ \\ duduardogeo@gmail.com \\ André Luiz Carvalho da Silva \\ Doutor em Geologia e Geofísica Marinha pela Universidade Federal Fluminense \\ Professor Adjunto do Departamento de Geografia, FFP/UERJ \\ andrelcsilvageouerj@gmail.com \\ Leonardo Rodrigues Eccard \\ Pós-Graduado em Dinâmicas Urbano-Ambientais e Gestão do Território, FFP/UERJ \\ leovascaum89@gmail.com \\ Carolina Pereira Silvestre \\ Doutoranda do Programa de Pós-graduação em Dinâmica dos Oceanos e da Terra, UFF \\ cps_silvestre@hotmail.com \\ Yame Bronze Medina Ramos \\ Mestranda do Programa de Pós-graduação em Dinâmica dos Oceanos e da Terra, UFF \\ yamemedina@hotmail.com
}

Artigo recebido em 05/11/2014 e aceito para publicação em 23/01/2015

http://dx.doi.org/10.12957/tamoios.2014.13511

Resumo

Abstract
As praias da enseada de Itaipu na Região Oceânica de Niterói, estado do Rio de Janeiro (Brasil), vêm sendo intensamente modificadas devido ao rápido processo de urbanização dessa região, principalmente a partir da década de 1970 com a construção da ponte Presidente Costa e Silva (Rio-Niterói). A crescente ocupação desse litoral se constitui no principal fator responsável pela degradação dos ecossistemas costeiros. A praia de Itaipu, apesar de ser a mais interna e, consequentemente a mais abrigada dentro da enseada de Itaipu, apresenta grande variabilidade na largura, em resposta as mudanças de curto prazo nas condições de mar. A faixa de areia da praia estreita-se de forma rápida em resposta a incidência de ondas de tempestades, restabelecendo-se logo a seguir quando do retorno às condições de tempo bom. As maiores variações foram constatadas na extremidade sul, devido à maior vulnerabilidade deste setor a incidência de ondas de tempestades. Os sedimentos da praia são constituídos predominantemente por areia quartzosa média, exceto em lâmina d'água de $5 \mathrm{~m}$ devido ao acúmulo de areia média com alto teor de carbonatos. Areia muito fina a fina aparece na profundidade de 7 metros, na área externa ao embasamento cristalino submerso.

Palavras-chave: Praia; dinâmica costeira; Itaipu.

The beaches of the Itaipu embayment in the Oceanic Region of Niterói, Rio de Janeiro state (Brazil), have been intensively modified due to the rapid urbanization of this area, especially from the 1970's, because of the construction of the President Costa e Silva bridge (RioNiterói). The increasing occupation of this coast constitutes the main factor responsible for the damage of coastal ecosystems. The Itaipu beach arc, despite being the innermost and consequently the most sheltered within the Itaipu embayment, shows great variability in width, in response to short-term changes in sea conditions. The beach width narrows rapidly in response to the incidence of stormy waves and it is immediately reestablished when good weather conditions return. The largest variations were observed at the south end, due to greater vulnerability of this sector to storm waves incidence. The beach sediments are basically composed by medium quartz-rich sorted sands, except at depth of $5 \mathrm{~m}$, due to the accumulation of medium sands with high concentrations of carbonates. Very fine and fine sands appear at a depth of 7 meters in the area outside the submerged crystalline basement.

Keywords: Beach; coastal dynamic; Itaipu. 


\section{INTRODUÇÃO}

O presente estudo objetivou identificar mudanças de curto prazo na morfologia da praia de Itaipu no município de Niterói, estado do Rio de Janeiro (Fig. 1), causadas pela variabilidade nas condições de mar em resposta a incidência de ondas de tempestades. Da mesma forma, procurou-se observar como se dá a recuperação desta praia após a ocorrência das ressacas. A aquisição de dados topobatimétricos e sedimentológicos permitiu a caracterização da morfologia emersa e submarina da praia, assim como, a ampliação do conhecimento acerca da dinâmica e susceptibilidade deste trecho do litoral de Niterói a eventos de alta energia.

A Região Oceânica de Niterói, desde o final da década de 1970, passou a vivenciar um intenso processo de ocupação, que se acentuou a partir da inauguração da Ponte Presidente Costa e Silva (Ponte Rio-Niterói) no ano de 1974, influenciando no rápido crescimento urbano da região. Esse processo ocasionou uma série de transformações na paisagem do litoral de Niterói. Desde então, este litoral vem sendo modificado por meio da destruição da vegetação de restinga, pela remoção de dunas e ocupação residencial das margens das lagoas de Piratininga e Itaipu. Problemas relativos à poluição por lixo e esgoto são também comuns e afetam as praias e lagoas do município. Diversos estudos foram realizados ao longo das últimas décadas no litoral estado do Rio de Janeiro, voltados para uma melhor compreensão da geomorfologia dos ambientes costeiros, com destaque para as abordagens relacionadas à dinâmica de praias (MUEHE, 1975; MUEHE, 1979; MUEHE \& CORRÊA, 1989; SILVA et al., 1999; SILVA, 2006; SILVA et al., 2009; ECCARD, 2014; entre outros).

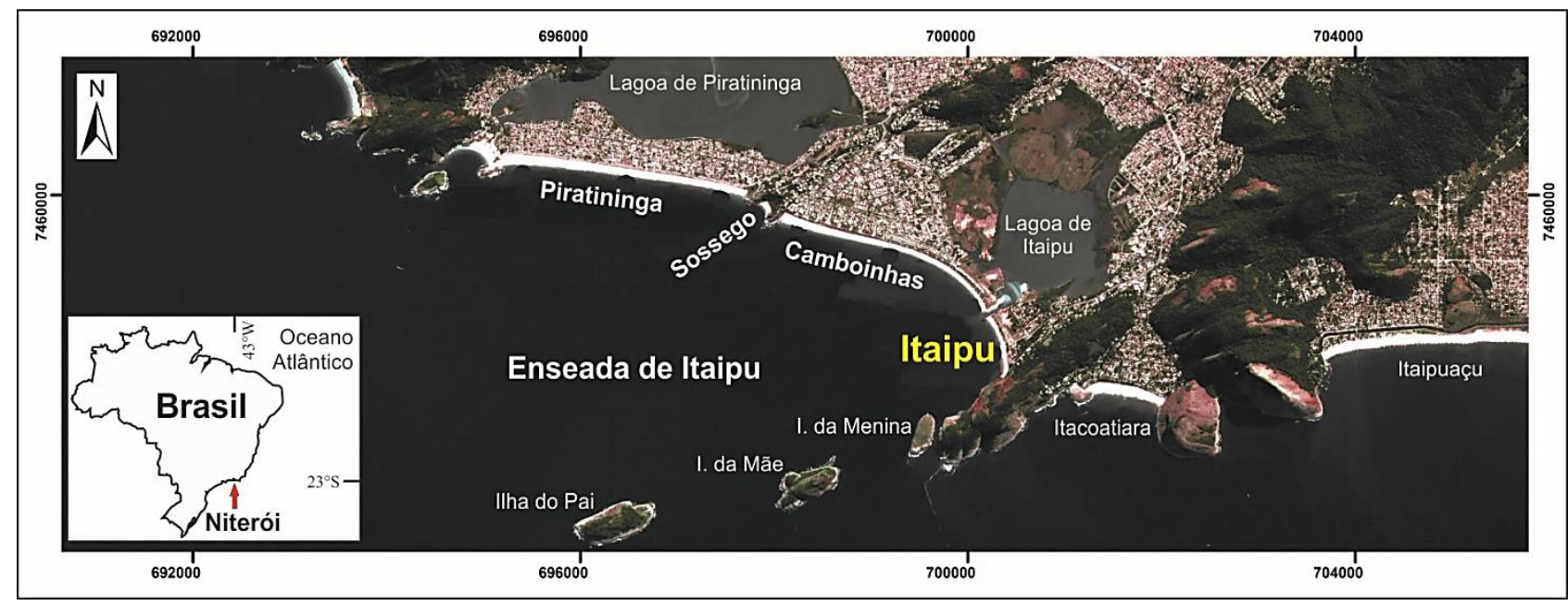

Figura 1 - Localização da enseada de Itaipu na região oceânica de Niterói, RJ.

\section{Área de estudo}

A praia de Itaipu encontra-se geograficamente localizada no extremo leste da enseada de Itaipu, num trecho do litoral de Niterói formado também pelas praias oceânicas de Piratininga, Sossego e Camboinhas (Fig. 1). A praia de Itaipu (Fig. 2) possui cerca de 800 metros de extensão e apresenta orientação aproximada norte-sul. Está limitada por um promontório rochoso ao sul, formado por gnaisse Pré-Cambriano (denominado Morro das Andorinhas); ao norte, limita-se com o canal que liga a Lagoa de Itaipu ao mar (Fig. 2).

Este litoral é caracterizado por uma maré do tipo mista, semidiurna, que não ultrapassa 1,5 metro (Diretoria de Hidrografia e Navegação da Marinha - DHN). As ondas representam o principal agente modelador deste litoral, juntamente com as correntes de deriva litorânea e de retorno. A praia de Itaipu está parcialmente protegida da incidência direta de ondas. As ondas provenientes de SE e S, normalmente associadas a condições de tempo bom, sofrem difração pelas ilhas do Pai, Mãe e Menina, que limitam a enseada de Itaipu (SILVA et al, 2009). As ondas de sudoeste, quase sempre formadas 
sob condições de tempestades, atingem o litoral durante as ressacas causando variações expressivas na largura e na morfologia da praia. A altura média das ondas neste litoral sob condições de tempo bom é de 1 metro, podendo atingir 3 metros durante a ocorrência de ressacas (MUEHE, 1979; SANTOS, 2001; SANTOS \& SILVA, 2004; SILVA et al., 2009). A região submarina adjacente a enseada de Itaipu apresenta um gradiente acentuado, com as profundidades próximas à costa aumentando rapidamente. Dados batimétricos da enseada de Itaipu, entre as isóbatas de 3 e $16 \mathrm{~m}$, apresentados por Salvador e Silva (2002), mostram um comportamento paralelo a linha de costa. Em profundidades superiores a $16 \mathrm{~m}$ a morfologia do fundo submarino é influenciada pelas ilhas do Pai, Mãe e Menina (Fig. 1). A morfologia de fundo da enseada de Itaipu é composta basicamente por areias médias e é controlada pelas ondas de tempestades. Os sedimentos mais finos tendem a se acumular a leste, trecho com baixa dinâmica, onde se localiza a praia de Itaipu (Figs. 1 e 2) (SALVADOR \& SILVA, 2002). Os sedimentos que chegam ao litoral são fornecidos em grande parte pela plataforma continental interna, devido à ausência de uma contribuição fluvial importante (OLIVEIRA \& MUEHE, 2013).

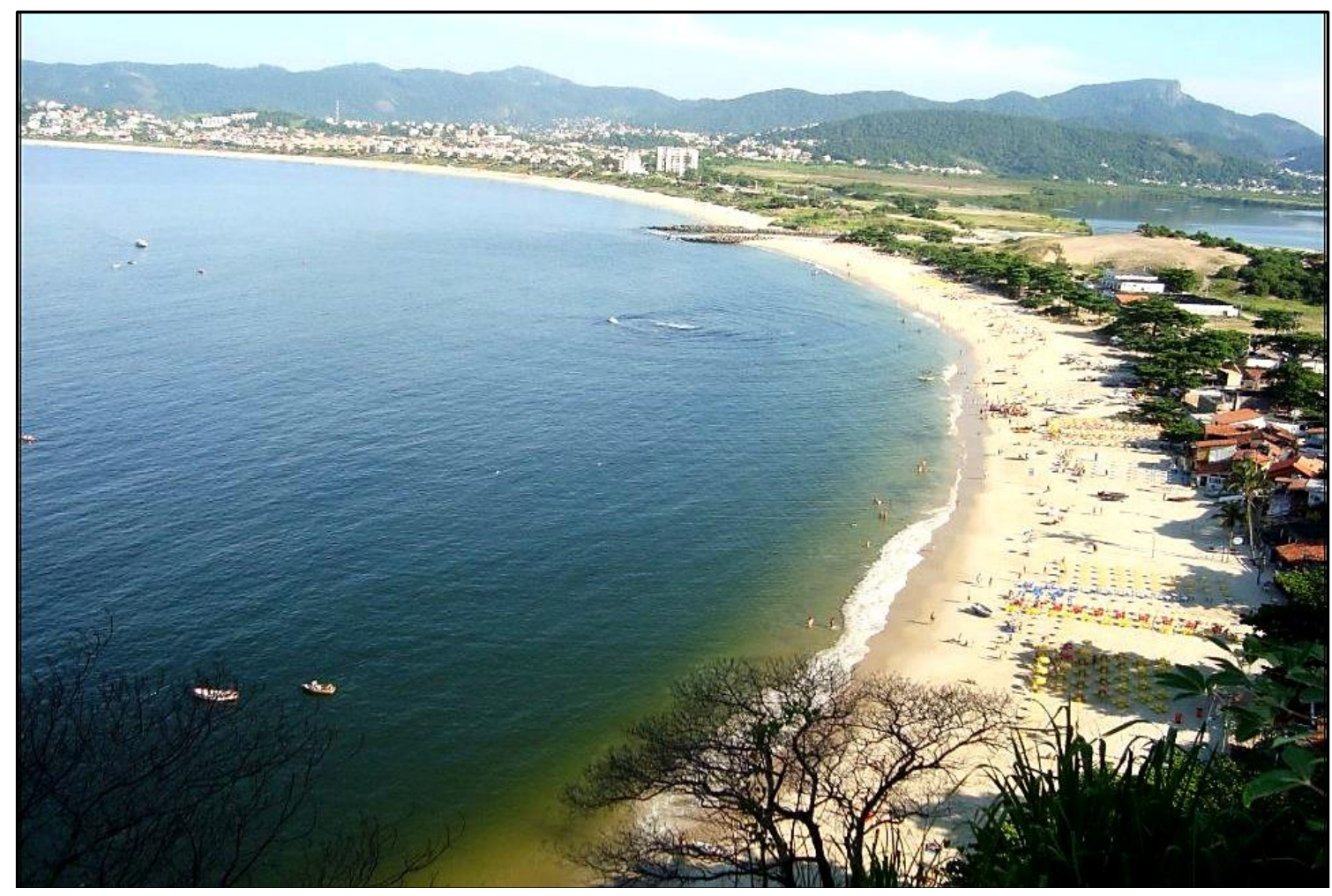

Figura 2 - Praia de Itaipu na região oceânica de Niterói. Foto: Leandro do Carmo, 2012.

\section{Materiais e Métodos}

As variações na largura da praia foram obtidas através de medições na porção emersa da mesma, nos três pontos de monitoramento (Fig. 3A): perfil A, no extremo norte da praia (S

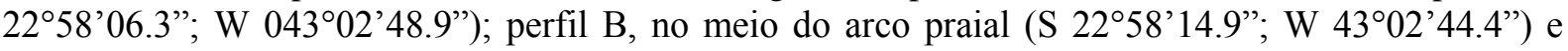
perfil C, no extremo sul da área de estudo (S $\left.22^{\circ} 58^{\prime} 23.7^{\prime \prime} ; \mathrm{W} 43^{\circ} 02^{\prime} 43.9^{\prime \prime}\right)$. As medições começaram no limite interno da praia, que neste caso coincide com o início da vegetação de restinga, indo até o alcance médio das ondas. Ao todo foram realizadas 36 medições entre os meses de fevereiro e julho de 2012, em intervalos que variam de 1 a 7 dias entre os monitoramentos.

O perfil topobatimétrico no meio do arco praial (área do perfil B - Fig. 3C) foi adquirido utilizando-se um equipamento constituído por nível topográfico acoplado a um tripé, mira (régua) e trena (Fig. 3B). A parte submarina do perfil foi realizada por uma equipe de três pessoas, sendo: duas na água, para medir a distância horizontal com a trena que estava amarrada a prancha e para alinhar a mira (Fig. 3C); e outra junto ao nível topográfico para registrar as medições das variações ao longo do 
perfil. Os locais de monitoramento foram devidamente georreferenciados com um GPS da marca Garmin 12 XL. O sistema de navegação usado foi o WGS 84.

Foram coletadas seis amostras de sedimentos na mesma área onde foi realizado o perfil topobatimétrico (Fig. 3A): duas na área emersa da praia e outras quatro na região submarina, abrangendo uma área representativa ao longo de todo o perfil estudado. A coleta dos sedimentos na região submarina foi realizada por meio de mergulho livre até a profundidade de 7 metros, com o uso de equipamento apropriado e sempre auxiliado por uma pessoa sobre a prancha.

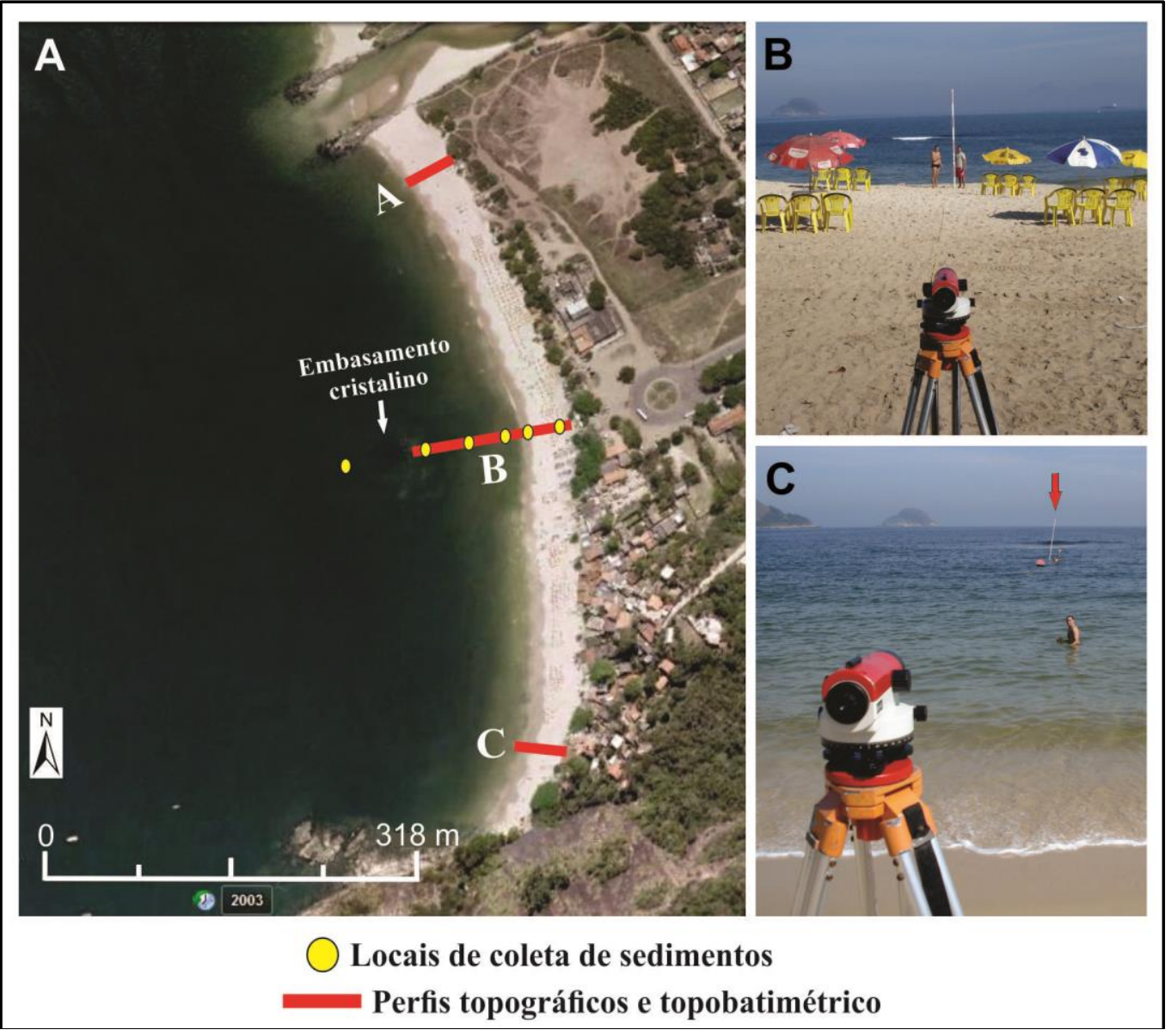

Figura 3 - Localização dos pontos de monitoramento e amostragem de sedimentos na porção emersa e submarina na praia de Itaipu (A). Levantamento topobatimétrico no meio do arco praial (B e C - a seta vermelha mostra a medição dentro d'água). Fotos: André Silva, 2012.

A análise granulométrica dos sedimentos foi baseada no método de peneiramento, utilizandose um conjunto de 7 peneiras $(4,00,2,00,1,00,0,500,0,250,0,125$ e 0,062 mm) para a separação das frações (Fig. 4A). Esta análise consistiu nas seguintes etapas: lavagem para a eliminação do sal, secagem na estufa a $50^{\circ} \mathrm{C}$, quarteamento, peneiramento e pesagem de cada fração (Fig. 4B). Os sedimentos foram classificados segundo Wentworth (1922) citado por Peittijohn (1975).

Para identificar o teor de carbonatos foram realizados diversos procedimentos laboratoriais: lavagem para a remoção de sais, secagem na estufa durante 24 horas a $110^{\circ} \mathrm{C}$ e pesagem de 20 gramas de material. Em seguida foi realizado o processo de descarbonatação a partir das seguintes etapas: acrescentou-se $30 \mathrm{ml}$ de ácido clorídrico diluído a 1N, as amostras são colocadas no agitador mecânico por 1 hora (Fig. 4C), em seguida acrescentou-se água destilada e algumas gotas do defloculante Cloreto de Cálcio $\left(\mathrm{CaCl}_{2}\right)$ (Fig. 4D), por fim a amostra foi centrifugada durante 5 minutos em 4.000 rpm (rotações por minuto), para a posterior extração do sobrenadante. Este procedimento é realizado sucessivas vezes até que não haja mais volatilização da amostra. Após a descarbonatação ocorre a 
Vulnerabilidade e variações de curto prazo da praia de Itaipu (Niterói-RJ) em resposta às mudanças nas condições de mar Eduardo de Macedo Rodrigues, André Luiz Carvalho da Silva, Leonardo Rodrigues Eccard, Carolina Pereira Silvestre e Yame

pesagem final da amostra depois de ter sido novamente seca na estufa.

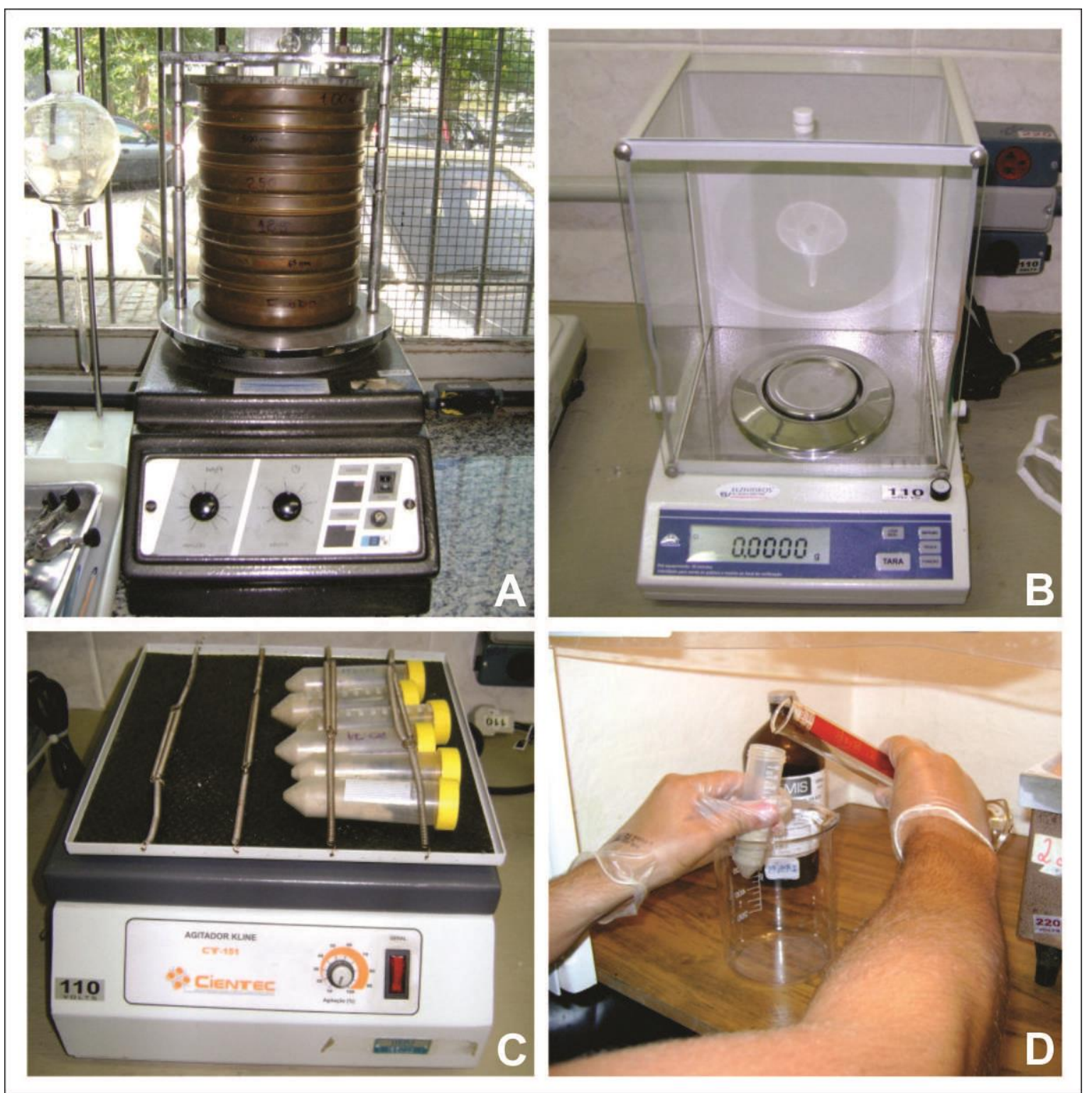

Figura 4 - Análise granulométrica dos sedimentos pelo método de peneiramento das frações (A e B) e análise do teor de carbonatos nos sedimentos coletados na porção emersa e submersa da praia de Itaipu (C e D). Fotos: Eduardo Rodrigues, 2012.

\section{Resultados e discussão}

A morfologia das praias localizadas na enseada de Itaipu é fortemente influenciada pela ação das ondas. A praia de Itaipu está parcialmente protegida da incidência direta de ondas devido a sua localização geográfica. As ondas provenientes do quadrante S, tanto aquelas normalmente associadas a condições de tempo bom quanto as de tempestades, sofrem difração pelas ilhas do Pai, Mãe e Menina, que limitam esta enseada. Ondas de sudoeste, normalmente associadas a condições de tempestades podem ultrapassar 2 metros de altura. Padrão semelhante foi constatado em estudos realizados anteriormente nas praias da enseada de Itaipu (SANTOS, 2001; SANTOS et al., 2004; SILVA et al., 2009). A forma de arrebentação das ondas varia, predominando o tipo progressiva (spilling), seguida por mergulhante (plunging) que ocorre quase sempre associada as ondas de tempestades. A arrebentação dessas ondas propicia a formação de uma estreita zona de surfe, que se apresenta mais desenvolvida na porção sul da praia próximo ao perfil C (Fig. 3A). A arrebentação de ondas no meio 
do arco praial (perfil B) ocorre mais afastada da praia (cerca de 70 metros). Isso ocorre devido à presença do embasamento cristalino submerso na porção submarina, que interfere na propagação das ondas forçando-as a arrebentar mais afastadas da praia (Fig. 3A).

As medições da largura da praia de Itaipu num curto período mostram que, apesar de geograficamente abrigada em um fundo de enseada, a mesma apresenta uma variabilidade morfológica considerável ao longo dos 800 metros de extensão, entre os meses de fevereiro e julho de 2012 (Tab. 1 e Fig. 5). As maiores variações na largura da praia foram observadas no extremo sul do arco praial (área do perfil C), que apresentou uma diferença de 38 metros entre o verão e inverno de 2012. Este trecho da praia também foi o mais estreito em relação às demais áreas ao longo de todo o período de monitoramento (Tab. 1 e Fig. 5), mostrando-se mais vulnerável as ondas de tempestades. Este setor da praia chegou a estreitar-se completamente em resposta a um evento de ressaca ocorrido nos dias 15 e 16 de junho, quando a faixa de areia da praia diminuiu quase 20 metros em 2 dias (Tab. 1 e Fig. 5).

Apesar da vulnerabilidade apresentada pela praia de Itaipu às ondas de tempestades, verificouse que a mesma se recupera de forma rápida logo após o período de influência das ondas de maior energia (Tab. 1 e Figs. 5 e 6). No extremo sul (perfil C), quase 30 metros de largura da faixa de areia da praia foram restabelecidos somente dois dias após o término de um evento de tempestades. A variabilidade morfológica apresentada pela praia parece ser uma resposta à dinâmica do próprio ambiente, com grandes mudanças nas condições hidrodinâmicas. $O$ estreitamento da mesma, em resposta a ação de ondas de tempestades, assim como, o seu alargamento subsequente quando predominam as ondas de tempo bom, ocorreu em diversos momentos ao longo dos monitoramentos (Tab. 1 e Figs. 5 e 6). Trata-se, portanto, de um comportamento clássico, verificado também em Itaipuaçu a leste da área de estudo, por Silva et al. (2008) e em Piratininga no setor oeste da enseada de Itaipu por Bastos \& Silva (1995), Santos et al. (2005) e Silva et al. (2009).

O perfil topobatimétrico realizado no meio do arco praial de Itaipu (perfil B - Fig. 3) possibilitou a caracterização morfológica da parte emersa e submarina da praia. Este perfil apresentou uma largura de 110 metros desde o limite interno da praia até o flanco interno do embasamento cristalino submerso, em lâmina d'água de 5 metros (Fig. 7). Os dados topobatimétricos mostram um pós-praia com cerca de 35 metros, ligeiramente inclinado para o oceano. A morfologia da frente de praia é mais íngreme (cerca de $25^{\circ}$ ) e mede apenas 15 metros. Já a face de praia, com $21^{\circ}$ de inclinação, é marcada pela presença de um banco arenoso na profundidade de $1,5 \mathrm{~m}$, e pela presença do embasamento cristalino Pré-Cambriano entre 5,5 e 7 metros de lâmina d'água. A presença do embasamento cristalino neste trecho do litoral do estado do Rio de Janeiro é marcante. Essas formações apresentam em geral uma topografia bastante irregular, formando diversos morros que podem alcançar mais de 700 metros de altitude, como é o caso do Corcovado (MANSUR et al., 2008). $\mathrm{Na}$ enseada de Itaipu o mesmo se apresenta acima do nível do mar limitando a enseada à leste (Morro das Andorinhas) e ao sul pelas ilhas do Pai, Mãe e Menina (SALVADOR \& SILVA, 2002). Em subsuperfície, o embasamento cristalino aparece desde 5-7 metros de profundidade até cerca de 1 metro abaixo do nível do mar, chegando quase a aflorar na maré baixa (Fig. 7). 
Vulnerabilidade e variações de curto prazo da praia de Itaipu (Niterói-RJ) em resposta às mudanças nas condições de mar Eduardo de Macedo Rodrigues, André Luiz Carvalho da Silva, Leonardo Rodrigues Eccard, Carolina Pereira Silvestre e Yame Bronze Medina Ramos

Tabela 1 - Dados de largura em metros dos 3 pontos de monitoramento na porção emersa da praia de Itaipu, registrados entre os meses de fevereiro e julho de 2012.

\begin{tabular}{cccc}
\hline Data & PA & PB & PC \\
\hline $08 / 02 / 2012$ & 42 & 46 & 31 \\
$09 / 02 / 2012$ & 34 & 36 & 29 \\
$16 / 02 / 2012$ & 40 & 43 & 31 \\
$23 / 02 / 2012$ & 44 & 48 & 31 \\
$01 / 03 / 2012$ & 41 & 45 & 27 \\
$05 / 03 / 2012$ & 43 & 48 & 29 \\
$07 / 03 / 2012$ & 48 & 53 & 38 \\
$09 / 03 / 2012$ & 47 & 51 & 36 \\
$15 / 03 / 2012$ & 46 & 47 & 32 \\
$17 / 03 / 2012$ & 39 & 40 & 19 \\
$19 / 03 / 2012$ & 46 & 47 & 32 \\
$21 / 03 / 2012$ & 45 & 50 & 33 \\
$23 / 03 / 2012$ & 44 & 51 & 35 \\
$30 / 03 / 2012$ & 30 & 28 & 16 \\
$02 / 04 / 2012$ & 43 & 42 & 26 \\
$13 / 04 / 2012$ & 45 & 46 & 33 \\
$16 / 04 / 2012$ & 41 & 38 & 27 \\
$18 / 04 / 2012$ & 42 & 43 & 33 \\
$24 / 04 / 2012$ & 34 & 35 & 14 \\
$27 / 04 / 2012$ & 35 & 37 & 22 \\
$07 / 05 / 2012$ & 48 & 51 & 36 \\
$15 / 05 / 2012$ & 29 & 32 & 20 \\
$19 / 05 / 2012$ & 47 & 48 & 33 \\
$22 / 05 / 2012$ & 44 & 51 & 37 \\
$26 / 05 / 2012$ & 41 & 43 & 28 \\
$14 / 06 / 2012$ & 41 & 44 & 22 \\
$20 / 06 / 2012$ & 50 & 48 & 33 \\
$29 / 06 / 2012$ & 39 & 38 & 18 \\
$08 / 07 / 2012$ & 40 & 37 & 24 \\
$11 / 07 / 2012$ & 44 & 43 & 25 \\
$13 / 07 / 2012$ & 34 & 37 & 23 \\
$14 / 07 / 2012$ & 31 & 33 & 19 \\
$16 / 07 / 2012$ & 23 & 28 & 0 \\
$17 / 07 / 2012$ & 24 & 28 & 9 \\
$18 / 07 / 2012$ & 46 & 45 & 29 \\
\hline & & & \\
\hline
\end{tabular}


Vulnerabilidade e variações de curto prazo da praia de Itaipu (Niterói-RJ) em resposta às mudanças nas condições de mar Eduardo de Macedo Rodrigues, André Luiz Carvalho da Silva, Leonardo Rodrigues Eccard, Carolina Pereira Silvestre e Yame Bronze Medina Ramos

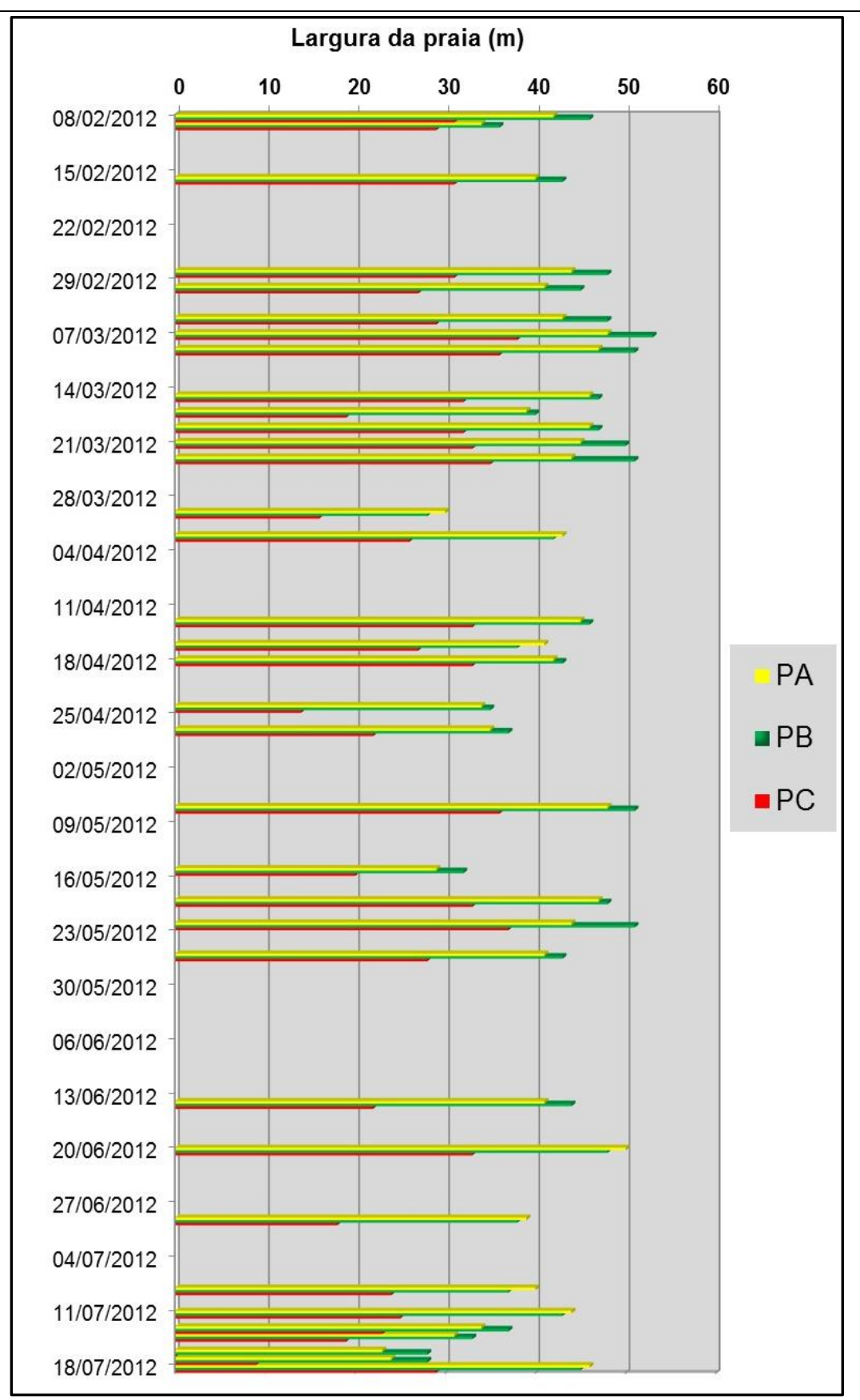

Figura 5 - Resultados das 36 medições na largura da faixa emersa da praia de Itaipu realizadas entre os meses de fevereiro e julho de 2012.

Os sedimentos da praia são constituídos basicamente por areia quartzosa média. Em lâmina d'água de 5 metros observou-se um acúmulo de areia média mal selecionada com alto teor de carbonatos $(63,59 \%$, ver Tab. 2), provavelmente, resultantes de carapaças de organismos oriundas do embasamento submerso. Na profundidade de 7 metros, na área externa ao embasamento cristalino submerso, predominam areias muito fina a fina (Fig. 7). Apesar da necessidade de se realizar um número maior de amostragens, percebe-se a influência expressiva do embasamento submerso no controle da sedimentação. A distribuição granulométrica dos sedimentos na porção submersa da praia apontada neste estudo, segue o mesmo padrão verificado por Salvador \& Silva (2002) para a porção leste da enseada de Itaipu, com a presença de areia média nos primeiros metros de profundidade, seguida por areia fina. 


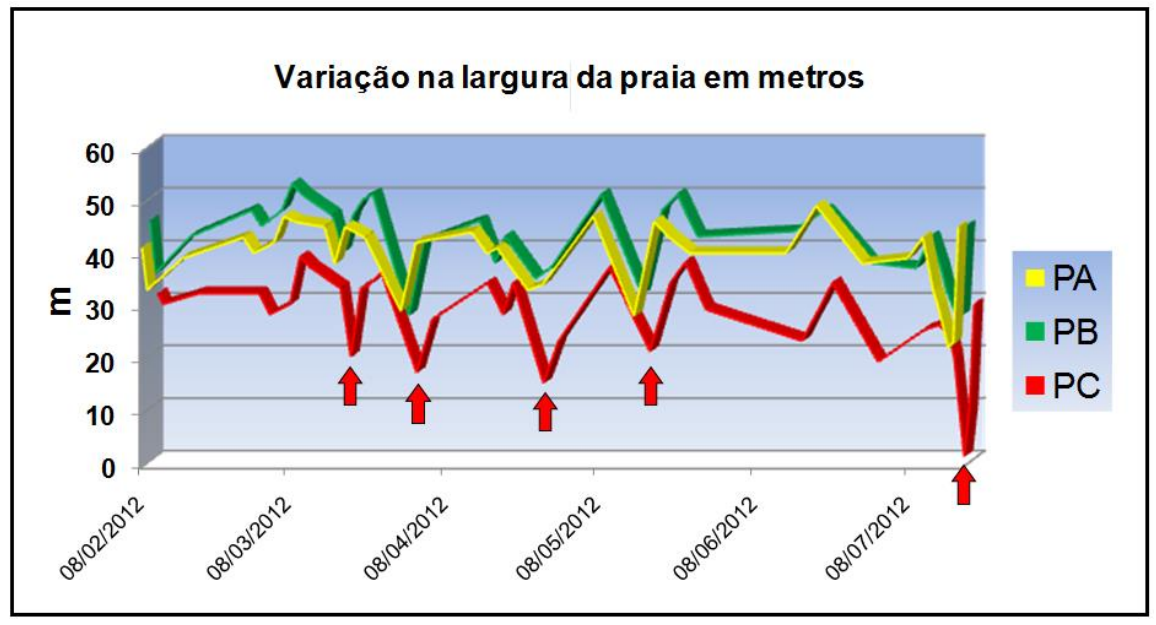

Figura 6 - Relação entre a variação na largura da praia de Itaipu e as ressacas ocorridas no período entre fevereiro a julho de 2012. As setas vermelhas indicam momentos de rápido estreitamento da praia em resposta à incidência de ondas de tempestades, seguidos por uma rápida recuperação.

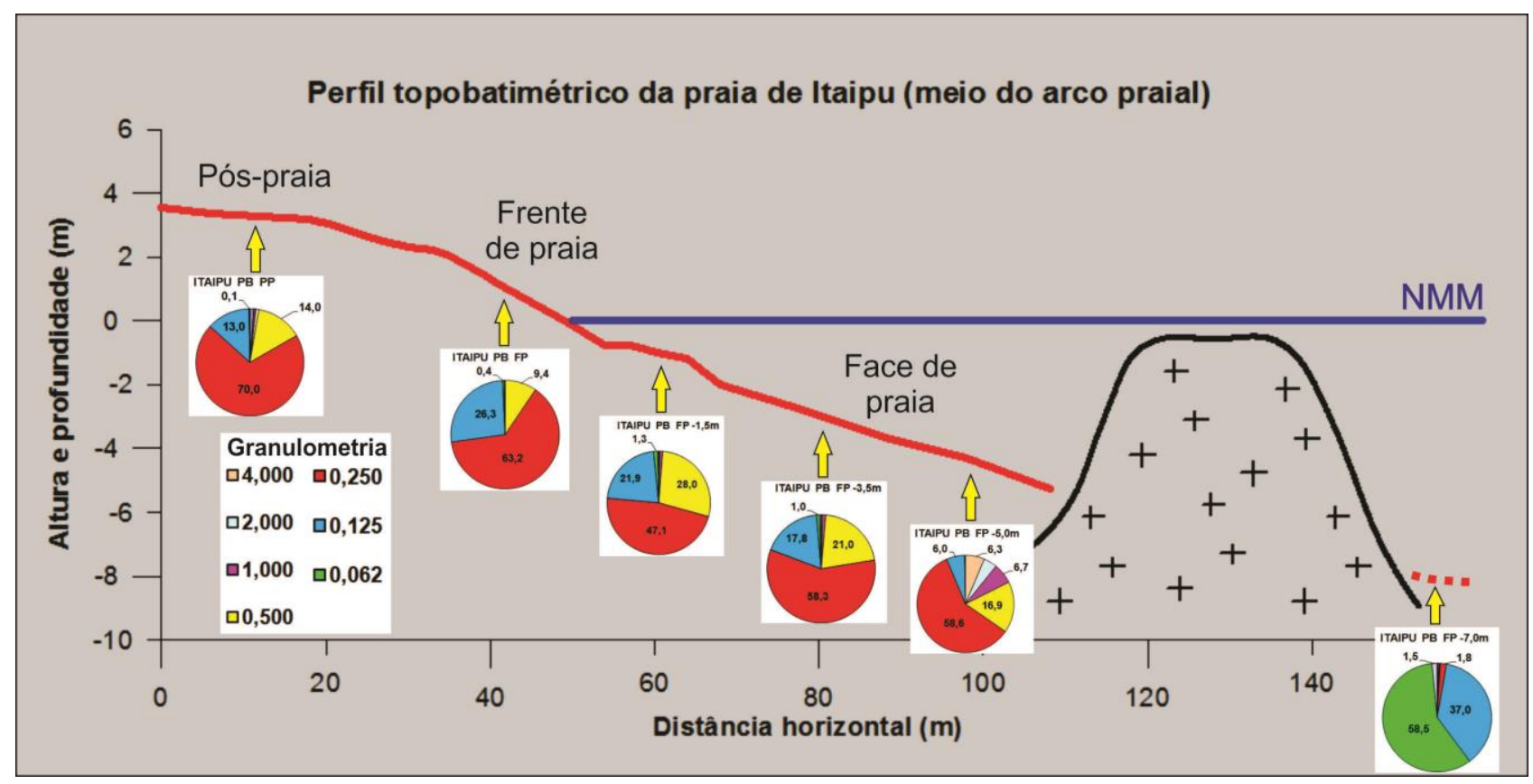

Figura 7 - Perfil topobatimétrico e granulometria dos sedimentos da praia de Itaipu. 
Vulnerabilidade e variações de curto prazo da praia de Itaipu (Niterói-RJ) em resposta às mudanças nas condições de mar Eduardo de Macedo Rodrigues, André Luiz Carvalho da Silva, Leonardo Rodrigues Eccard, Carolina Pereira Silvestre e Yame Bronze Medina Ramos

Tabela 2 - Resultado das análises granulométricas e do teor de carbonatos dos sedimentos coletados na porção emersa e submersa da praia de Itaipu.

\begin{tabular}{|c|c|c|c|c|c|c|c|c|c|c|}
\hline \multirow[t]{2}{*}{ Amostra } & \multicolumn{8}{|c|}{ Granulometria (\%) } & \multicolumn{2}{|c|}{$\begin{array}{c}\text { Composição } \\
(\%)\end{array}$} \\
\hline & 4,00 & 2,00 & 1,00 & 0,50 & 0,25 & 0,125 & 0,062 & Lama & $\mathrm{SiO}_{2}$ & $\mathrm{CaCO}_{3}$ \\
\hline $\mathrm{PP}$ & 1,10 & 0,74 & 0,87 & 14,00 & $\mathbf{7 0 , 0 0}$ & 13,03 & 0,13 & 0,13 & 97,07 & 2,93 \\
\hline FP & 0,00 & 0,00 & 0,06 & 9,37 & 63,20 & 26,32 & 0,43 & 0,33 & 95,85 & 4,15 \\
\hline$-1,5$ & 0,00 & 0,30 & 0,95 & 28,04 & 47,14 & 21,93 & 1,28 & 0,32 & 90,17 & 9,83 \\
\hline$-3,5$ & 0,00 & 0,32 & 1,03 & 21,00 & 58,25 & 17,80 & 0,95 & 0,45 & 86,76 & 13,09 \\
\hline$-5,0$ & 6,26 & 4,45 & 6,73 & 16,92 & $\mathbf{5 8 , 5 8}$ & 6,00 & 0,20 & 0,10 & 35,41 & 63,59 \\
\hline$-7,0$ & 0,09 & 0,28 & 0,32 & 0,26 & 1,80 & 37,01 & 58,45 & 1,53 & 92,98 & 7,02 \\
\hline
\end{tabular}

A marcação em negrito indica a fração e composição predominante em cada amostra. PP, pós-praia; FP, frente de praia; -1,5 a -7,0, profundidade em metros.

\section{Conclusões}

A praia de Itaipu apresentou variações morfológicas ao longo de toda a sua extensão em resposta às mudanças repentinas nas condições de mar. A variação na largura da praia foi mais expressiva na extremidade sul (perfil C). Este mesmo setor do arco praial apresentou maior vulnerabilidade à incidência de ondas de tempestades, chegando a estreitar-se completamente durante a ocorrência de ressacas no inverno de 2012. No entanto, a praia mostrou uma boa capacidade de recuperação, alargando-se rapidamente após a passagem de ressacas. Os sedimentos que formam a praia são constituídos principalmente por areia quartzosa média, tanto na porção emersa quanto submarina, com exceção da área localizada após o flanco externo do embasamento, onde predomina areia muito fina a fina.

Contudo, pode-se concluir que as praias, mesmo quando abrigadas em fundo de enseadas, podem apresentar problemas decorrentes da incidência direta de ondas de tempestades. Tais problemas podem estar associados a perda de estruturas de engenharia construídas dentro dos limites da praia, remoção da vegetação e dunas na retaguarda da mesma, entre outros. O entendimento da dinâmica da praia de Itaipu, assim como dos vários ambientes localizados ao longo da costa, é de grande relevância para o gerenciamento dessas áreas, visando à preservação dos ecossistemas costeiros e a diminuição dos riscos de perdas e danos à sociedade.

\section{Agradecimentos}

O presente estudo integra resultados obtidos ao longo de projetos desenvolvidos junto ao Departamentos de Geologia da UFF e de Geografia da UERJ-FFP, voltados para o entendimento da dinâmica do litoral de Niterói. Agradecimentos à UERJ e à CAPES pela concessão das bolsas aos estudantes de graduação e mestrado, respectivamente. Agradecemos também ao Laboratório de Geociências da FFP-UERJ que disponibilizou a estrutura necessária para a realização das análises nos sedimentos. 


\section{Referências Bibliográficas}

BASTOS, A. C. \& SILVA, C. G. 1995. Study of morphological variations from beach profiles in Piratininga Beach, Niterói -RJ-Brazil. Anais V Congresso da ABEQUA, p. 205-210.

DHN, Folha de Bordo, FB - 1500-001/79. Diretoria de Hidrografia e Navegação da Marinha do Brasil, 1980.

ECCARD, L.R. 2014. Dinâmica das praias oceânicas de Niterói (RJ) e vulnerabilidade às ondas de tempestades. Monografia do Curso de Especialização em Dinâmicas Urbano-ambientais e Gestão do Território da UERJ-FFP. pp. 62.

MANSUR, K. L., CARVAlHO, I. S., DELPHIM, C. F. M. BARROSO, E. V. 2008. O gnaisse facoidal: a mais carioca das rochas. Anuário do Instituto de Geociências - UFRJ. 31(2). pp. 922.

MUEHE, D. C. E. H. 1975. Análise Ambiental no Sistema Costeiro Sul-oriental do Estado do Rio de Janeiro. Dissertação de Mestrado. Pós Graduação em Geografia da Universidade Federal do Rio de Janeiro. pp. 141.

MUEHE, D. C. E. H. 1979. Sedimentology and Topography of a High Energy Coastal Environment Between Rio de Janeiro and Cabo Frio - Brazil. Anais da Academia Brasileira de Ciências. 51 (3), pp. 473-481.

MUEHE, D. C. E. H \& CORRÊA, C. H. T. 1989. The Coastline Between Rio de Janeiro and Cabo Frio. Coastlines of Brazil. American Society of Civil Engineers. New York, pp. 110- 123.

OLIVEIRA, J. F. \& MUEHE, D. C. E. H. 2013. Identificação de áreas de sedimentos compatíveis na plataforma continental interna para recuperação de praias entre as cidades de Niterói e Macaé Rio de Janeiro, Brasil. Revista da Gestão Costeira Integrada, 13 (1), pp. 89-99.

PETTIJOHN, F. J. 1975. Sedimentary Rocks. Third Edition. Harper and Row Publishers. pp. 68-81.

SALVADOR, M. V. S. \& SILVA, M. A. M. 2002. Morphology and sedimentology of the Itaipu Embayment - Niterói/RJ. Anais da Academia Brasileira de Ciências. p. 127 - 134.

SANTOS, C. L. 2001. Dinâmica Sazonal e os Efeitos das Ressacas nas Praias de Niterói (Rio de Janeiro). Dissertação de Mestrado. Programa de Pós Graduação em Geologia e Geofísica Marinha da Universidade Federal Fluminense. pp.151.

SANTOS, C. L. dos, SILVA, M. A. M. e SALVADOR, M. V. S. 2004. Dinâmica Sazonal e os Efeitos das Ressacas nas Praias de Niterói/RJ. Revista Brasileira de Geociências. pp. 355-360.

SILVA, M. A. M., RESENDE, M. C. C. M. \& SANTOS, C. L. 1999. Um Estudo sobre a Dinâmica das Praias de Niterói (Baía de Guanabara, RJ). Anais da Academia Brasileira de Ciências. N ${ }^{\circ}$ 71 (4) pp. $962-967$.

SILVA, A. L. C. 2006. Comportamento Morfológico e Sedimentológico do Litoral de Itaipuaçú (Maricá) e Piratininga (Niterói), RJ, nas últimas três décadas. Dissertação de Mestrado. Programa de Pós Graduação em Geologia e Geofísica Marinha da Universidade Federal Fluminense.

SILVA, A. L. C.; SILVA, M. A. M.; SANTOS, C. L., 2008. Comportamento morfológico e sedimentar da praia de Itaipuaçú (Maricá, RJ) nas últimas três décadas. Revista Brasileira de Geociências. 38(1), p. 89/99

SILVA, A. L. C., SILVA, M. A. M \& SANTOS, C. L. 2009. Morfodinâmica e a estabilidade da praia de Piratininga, Niterói (RJ). Revista Brasileira de Geociências. 39(4), pp. 684 - 693. 